\title{
Prectureric

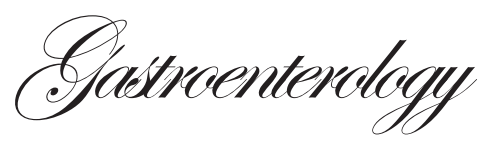 \\ Utility of Tc99m-Mebrofenin hepato-biliary scintigraphy (HIDA scan) for the diagnosis of biliary atresia
}

\author{
Ira Shah, ${ }^{1}$ Sushmita Bhatnagar, ${ }^{1}$ Venkatesh Rangarajan, ${ }^{2}$ Nikhil \\ Patankar $^{1}$
}

\section{ABSTRACT}

Pediatric Hepatobiliary Clinic ${ }^{1}$ and Department of Radionuclide Medicine, ${ }^{2}$

BJ Wadia Hospital for Children,

Tata Hospital

Mumbai, India

Correspondence:

Dr. Ira Shah

Email:irashah@pediatriconcall.com
Aim: To determine the utility of Tc99m-Mebrofenin hepato-biliary scintigraphy (HIDA scan) for diagnosis of biliary atresia in patients with neonatal cholestasis.

Methods: Our study involves the retrospective analysis of 46 patients with neonatal cholestasis who underwent HIDA scans at the Pediatric Hepatobiliary Clinic, BJ Wadia Hospital for Children from May 2005 to July 2007. Biliary atresia (BA) was diagnosed on the basis of intra-operative cholangiogram. Non-BA patients were included in the neonatal hepatitis (NH) group. All patients received phenobarbitone and ursodeoxycholic acid for 5 days, prior to the HIDA scan. The HIDA scan was evaluated on the basis of uptake of the radioactive tracer by the liver at 5 minutes after intravenous injection; retention of radioactive tracer within the liver at 24 hours after injection and visualization of excretion of tracer into the intestine upto 24 hours after administration. The results of the HIDA scans were analyzed and correlated with the final diagnosis, gender and age of the patients. Chi-square test was employed for statistical analysis.

Results: The age of presentation of our patients ranged from 5 days to 6 months. The male: female ratio was 37:9. Of the total 46 patients, 28 had BA and 18 had NH. All $28(100 \%)$ patients diagnosed with BA showed persistent radiotracer in the liver at 24 hours whereas $17(94.4 \%)$ of the $18 \mathrm{NH}$ patients showed hepatic radiotracer retention $(\mathrm{p}=0.207)$, the difference being statistically insignificant. Twenty two (78.6\%) patients of BA showed no excretion of the radiotracer at 24 hours whereas only $7(38.9 \%)$ of the $\mathrm{NH}$ group did not excrete the radiotracer $(\mathrm{p}=0.007)$, which was statistically significant. Neither the sex nor the age of the child contributed to any difference on the hepatic retention ( $\mathrm{p}=0.618$ and 0.235 , respectively) or on the intestinal excretion ( $\mathrm{p}=0.307$ and 0.9 , respectively) of the radiotracer.

Conclusion: HIDA scan is a useful tool for screening of biliary atresia in patients with neonatal cholestasis. Non excretion of the radioactive radiotracer into the intestines even after 24 hours of radiotracer administration can suggest biliary atresia in majority of patients.

KEYWORDS: HIDA scan, biliary atresia, neonatal hepatitis, Tc99m-Mebrofenin

\section{Introduction}

Biliary atresia is characterized by obliteration or discontinuity of the extra hepatic biliary system, resulting in obstruction to bile flow. ${ }^{1}$ The disorder represents the most common surgically treatable cause of cholestasis encountered during the newborn 
period. If not surgically corrected, secondary biliary cirrhosis invariably results. Thus early diagnosis of biliary atresia remains imperative and liver biopsy and/or intra-operative cholangiogram remains the gold standard within the diagnostic modalities used to confirm biliary atresia. Studies have demonstrated $100 \%$ sensitivity of Tc99m-Mebrofenin hepatobiliary scintigraphy (HIDA scan) for picking up biliary atresia, however its specificity has been reported to range from 88.6 to $92 \% .{ }^{2,3}$ We undertook a study to determine the utility of HIDA scan for diagnosing biliary atresia in patients with clinical evidence of neonatal cholestasis, and to determine whether this non-invasive imaging test is a useful tool for distinguishing biliary atresia from other causes of cholestasis such as neonatal hepatitis.

\section{Methods}

This retrospective analysis was undertaken on patients treated at our center from May 2005 to July 2007. Infants less than 6 months of age suffering from jaundice with high colored urine (conjugated hyperbilirubinemia), with an onset before 3 months of age were included in the study. Patients were classified into 3 age groups: $<1$ month, 1-3 months and $>3$ months. A detailed clinical history was elucidated and recorded for each patient, followed by a thorough clinical examination. To determine the etiology of neonatal cholestasis, TORCH titres, urine for reducing substances, and intra-operative cholangiogram with liver biopsy were investigated. Additional tests including serum alpha- 1 antitrypsin, urine aminoacidogram, urine organic acids, and echocardiography were done as and when required. In addition, the patients were tested for liver function tests, and ultrasonography of the abdomen and stool for stercobilinogen.

All these patients underwent HIDA scan prior to which they received phenobarbitone at a dose of $5 \mathrm{mg} / \mathrm{kg} / \mathrm{d}$ for 5 days and ursodeoxycholic acid at $15 \mathrm{mg} / \mathrm{kg} /$ day. HIDA scan was performed on each patient after intravenous administration of Tc99m-Mebrofenin into a peripheral vein, followed by serial static images of the abdomen-pelvis in both anterior and posterior views, of at least $500 \mathrm{~K}$ counts, using a dual headed gamma camera. Images were acquired after 5 minutes, 30 minutes, 2 hours, 4 hours and 24 hours of radiotracer administration. The images were evaluated for uptake of the radioactive tracer by the liver at 5 minutes after intravenous injection, retention of radioactive tracer within the liver at 24 hours after injection and visualization of excretion of tracer into the intestine upto 24 hours after administration. The HIDA scans were read by an experienced nuclear physician. Biliary atresia was diagnosed on absence of radiotracer excretion on intra-operative cholangiography. All patients without biliary atresia or a surgical cause of neonatal cholestasis were classified under the neonatal hepatitis group. Correlation of radiotracer excretion on HIDA scan was drawn with each patient's final diagnosis, by an intra-operative cholangiogram. Factors such as age and sex affecting uptake and excretion of the radiotracer were also studied. Statistical analysis was carried out using the Chi-square test and a $\mathrm{p}$ value of $<0.05$ was taken as significant.

\section{Results}

Forty six infants with neonatal cholestasis were included in this study, of which 36 (78.3\%) were males and $10(21.7 \%)$ females. The age of our patients at presentation ranged from 7 days to 6 months with the median being 2 months and mean age being $2.29 \pm 0.204$ months. Five (10.9\%) patients were in the $<1$ month group of which 4 were males and 1 female; 33 (71.7\%) patients were in the 1-3 months age group of which 27 were males and 7 females. Eight $(17.4 \%$ ) patients were $>3$ months of age, of which 5 were males and 3 females. Twenty eight $(60.9 \%)$ patients were diagnosed to have biliary atresia. All these 28 patients $(100 \%)$ showed persistent hepatic retention of the radiotracer at 24 hours, and 22 (78.6\%) of these 28 patients did not demonstrate any intestinal excretion of radiotracer up to 24 hours, On the other hand, of the 18 patients in the neonatal hepatitis group, 17 (94.4\%) showed persistent hepatic retention of the radiotracer and $7(38.9 \%)$ showed no intestinal excretion of the radiotracer at 24 hours $(\mathrm{p}=0.007)$.

Of the 36 boys, 14 (38.9\%) showed intestinal radiotracer excretion and of the 10 girls, $3(30 \%)$ excreted the radiotracer into the intestine $(\mathrm{p}=0.606)$. One boy $(2.78 \%)$ did not show persistent retention of the radiotracer in the liver, whereas all $10(100 \%)$ girls showed persistent hepatic retention of the

Table 1: Uptake and excretion of radiotracer on HIDA scan in various age groups

\begin{tabular}{llll}
\hline Age group & $\begin{array}{l}\text { Persistent retention } \\
\text { of radiotracer in the } \\
\text { liver at 24 hours }\end{array}$ & $\begin{array}{l}\text { Visualization of } \\
\text { excretion of } \\
\text { radiotracer into } \\
\text { intestine at } \\
\mathbf{2 4} \text { hours }\end{array}$ & Total \\
\hline$<1$ months & $5(100 \%)$ & $2(40 \%)$ & 5 \\
$1-3$ months & $32(97 \%)$ & $12(36.4 \%)$ & 33
\end{tabular}


radiotracer $(\mathrm{p}=0.594)$. Uptake and excretion of the radiotracer in various age groups is depicted in Table 1.

\section{Discussion}

Incidence of biliary atresia ranges from 1 in 10,000-15,000 live births in various studies and its incidence is reported highest in Asian population. Liver biopsy and intra-operative cholangiogram remain the gold standards for diagnosing biliary atresia. Also no single biochemical test accurately discriminates between biliary atresia and other causes of neonatal cholestasis. ${ }^{1}$ HIDA scan has been used as a screening tool in tandem with clinical data for the diagnosis of biliary atresia as demonstrated in previous studies. ${ }^{2-5}$ Non-excretion of the radioactive radiotracer into the intestines at 24 hours is highly suggestive of biliary atresia and was found statistically significant in our study. However absence of gut excretion on a HIDA scan does not necessarily indicate extra-hepatic obstruction and such non-draining scans can also be seen in severe neonatal hepatitis or presence of interlobular bile duct paucity. ${ }^{2,45}$ Similarly in our study, 7 patients (38.9\%) with neonatal hepatitis did not show any excretion of the radiotracer on liver scan. In fact, Spivak et $\mathrm{al}^{2}$ have found on repeated scanning that initial non-excretion of radiotracer could show excretion of radiotracer in children with neonatal hepatitis on subsequent scans. In our patients with neonatal hepatitis who showed no excretion of radiotracer, we did not undertake repeat scanning as these patients underwent a liver biopsy and biliary atresia was ruled out in them. This test has been associated with a $10 \%$ rate of false positive and false negative diagnostic errors. ${ }^{1}$ In our study also $21.4 \%$ of patients with biliary atresia excreted the radiotracer on HIDA scan, whereas $38.9 \%$ of patients with neonatal hepatitis did not show any excretion of the radiotracer. We also did not find any correlation between sex and age of the patient and excretion of the radiotracer in our study.

Early diagnosis of biliary atresia is essential to prevent the development of further complications like liver cell failure and cirrhosis and thereby HIDA scan as a non-invasive modality can be a very useful screening method to differentiate biliary atresia from neonatal hepatitis. However liver biopsy or intraoperative cholangiogram still is needed for confirmation.

\section{References}

1. Schwarz SM. Biliary Atresia. Available on url: http:// www.emedicine.com/ped/topic237.htm . Accessed on 6th May 2008.

2. Spivak W, Sarkar S, Winter D, Glassman M, Donlon E, Tucker KJ. Diagnostic utility of hepatobiliary scintigraphy with $99 \mathrm{mTc}-$ DISIDA in neonatal cholestasis. J Pediatr. 1987;110:855-61.

3. Poddar U, Bhattacharya A, Thapa BR, Mittal BR, Singh K. Ursodeoxycholic acid-augmented hepatobiliary scintigraphy in the evaluation of neonatal jaundice. J Nucl Med. 2004;45:1488-92.

4. Johnson K, Alton HM, Chapman S. Evaluation of mebrofenin hepatoscintigraphy in neonatal-onset jaundice. Pediatr Radiol. 1998;28:937-41.

5. Gilmour SM, Hershkop M, Reifen R, Gilday D, Roberts EA. Outcome of hepatobiliary scanning in neonatal hepatitis syndrome. J Nucl Med. 1997;38:1279-82. 